\title{
Generalization of the Levinson inequality with applications to information theory
}

\author{
Muhammad Adeel ${ }^{1 *}$ (D) Khuram Ali Khan ${ }^{1}$, Đilda Pečarić ${ }^{2}$ and Josip Pečarić ${ }^{3}$
}

\section{"Correspondence:}

adeel.uosmaths@gmail.com

'Department of Mathematics,

University of Sargodha, Sargodha,

Pakistan

Full list of author information is

available at the end of the article

\begin{abstract}
In the presented paper, Levinson's inequality for 3-convex functi 7 is generalked by using two Green's functions. Čebyšev, Grüss, and Ostrowski- /pe w bolnds are found for the functionals involving data points of two tynes. reover, the main results are applied to information theory via f-diverge -e, Rény ‘ vergence, Rényi entropy, Shannon entropy, and Zipf-Mandelbrot la v.
\end{abstract}

Keywords: Levinson's inequality; Information ur ry

\section{Introduction and preliminaries}

In [12], Ky Fan's inequality is ge lized by Levinson for 3-convex functions as follows:

Theorem A Let $f: I=(f, 2 \alpha)-\mathbb{p} u$ th $f^{(3)}(t) \geq 0$. Let $x_{k} \in(0, \alpha)$ and $p_{k}>0$. Then

$$
J_{1}(f) \geq 0
$$

where

$$
\begin{aligned}
& J_{1}\left(f \left(\frac{1}{\mathbf{P}_{n}} \sum_{\rho=1}^{n} p_{\rho} f\left(2 \alpha-x_{\rho}\right)-f\left(\frac{1}{\mathbf{P}_{n}} \sum_{\rho=1}^{n} p_{\rho}\left(2 \alpha-x_{\rho}\right)\right)-\frac{1}{\mathbf{P}_{n}} \sum_{\rho=1}^{n} p_{\rho} f\left(x_{\rho}\right)\right.\right. \\
& +f\left(\frac{1}{\mathbf{P}_{n}} \sum_{\rho=1}^{n} p_{\rho} x_{\rho}\right) .
\end{aligned}
$$

Working with the divided differences, the assumptions of differentiability on $f$ can be weakened.

In [18], Popoviciu noted that (1) is valid on $(0,2 a)$ for 3-convex functions, while in [2], Bullen gave a different proof of Popoviciu's result and also the converse of (1).

Theorem B (a) Let $f: I=[a, b] \rightarrow \mathbb{R}$ be a 3-convex function and $x_{n}, y_{n} \in[a, b]$ for $n=$ $1,2, \ldots, k$ such that

$$
\max \left\{x_{1} \ldots x_{k}\right\} \leq \min \left\{y_{1} \ldots y_{k}\right\}, \quad x_{1}+y_{1}=\cdots=x_{k}+y_{k}
$$


and $p_{n}>0$. Then

$$
J_{2}(f) \geq 0,
$$

where

$$
J_{2}(f(\cdot))=\frac{1}{\mathbf{P}_{k}} \sum_{\rho=1}^{k} p_{\rho} f\left(y_{\rho}\right)-f\left(\frac{1}{\mathbf{P}_{k}} \sum_{\rho=1}^{k} p_{\rho} y_{\rho}\right)-\frac{1}{\mathbf{P}_{k}} \sum_{\rho=1}^{k} p_{\rho} f\left(x_{\rho}\right)+f\left(\frac{1}{\mathbf{P}_{k}} \sum_{\rho=1}^{k} p_{\rho} x_{\rho}\right) .
$$

(b) Iff is continuous and $p_{\rho}>0,(4)$ holds for all $x_{\rho}, y_{\rho}$ satisfying (3), then $f$ is 3-c nvex.

In [17], Pečarić weakened assumption (3) and proved that inequality (1) st. olds, 1.e., the following result holds:

Theorem C Letf $: I=[a, b] \rightarrow \mathbb{R}$ be a 3-convex function, $p_{k}>0$, a let for $k=1, \ldots, n, x_{k}$, $y_{k}$ be such that $x_{k}+y_{k}=2 \breve{c}, x_{k}+x_{n-k+1} \leq 2 \breve{c}$ and $\frac{p_{k} x_{k}+p_{n-k+1} x_{n-n}}{p_{k}+p_{n-k+1}}$

In [15], Mercer made a notable work by replacing the ( $0_{2}$ on of symmetric distribution of points $x_{i}$ and $y_{i}$ with symmetric variances of points $c_{i}$ and $y_{i}$. The second condition is a weaker condition.

Theorem D Let $f$ be a 3-convex func ion on $\mathrm{L}$. '1] $p_{k}$ be positive such that $\sum_{k=1}^{n} p_{k}=1$. Also let $x_{k}, y_{k}$ satisfy (3) and

$$
\sum_{\rho=1}^{n} p_{\rho}\left(x_{\rho}-\sum_{\rho=1}^{n} p_{\rho} x_{\rho}\right)^{2}=\sum_{\rho=1}\left(y_{\rho}-\sum_{\rho=1}^{n} p_{\rho} y_{\rho}\right)^{2} .
$$

\section{Then (1) holds.}

On the er hald, the error function $e_{\mathcal{F}}(t)$ can be represented in terms of the Green's funct: $G_{\mathcal{J}}(+s)$ of the boundary value problem

$$
\begin{aligned}
& z^{(n)}(t)=0 \\
& z^{\prime}\left(a_{1}\right)=0, \quad 0 \leq i \leq p \\
& z^{(i)}\left(a_{2}\right)=0, \quad p+1 \leq i \leq n-1, \\
& e_{F}(t)=\int_{a_{1}}^{a_{2}} G_{F, n}(t, s) f^{(n)}(s) d s, \quad t \in[a, b],
\end{aligned}
$$

where

$$
G_{F, n}(t, s)=\frac{1}{(n-1) !} \begin{cases}\sum_{i=0}^{p}\left(\begin{array}{c}
n-1 \\
i
\end{array}\right)\left(t-a_{1}\right)^{i}\left(a_{1}-s\right)^{n-i-1}, & a_{1} \leq s \leq t \\
-\sum_{i=p+1}^{n-1}\left(\begin{array}{c}
n-1 \\
i
\end{array}\right)\left(t-a_{1}\right)^{i}\left(a_{1}-s\right)^{n-i-1}, & t \leq s \leq a_{2} .\end{cases}
$$

The following result holds in [1]: 
Theorem E Let $f \in C^{n}[a, b]$, and let $P_{F}$ be its 'two-point right focal' interpolating polynomial. Then, for $a \leq a_{1}<a_{2} \leq b$ and $0 \leq p \leq n-2$,

$$
\begin{aligned}
f(t)= & P_{F}(t)+e_{F}(t) \\
= & \sum_{i=0}^{p} \frac{\left(t-a_{1}\right)^{i}}{i !} f^{(i)}\left(a_{1}\right) \\
& +\sum_{j=0}^{n-p-2}\left(\sum_{i=0}^{j} \frac{\left(t-a_{1}\right)^{p+1+i}\left(a_{1}-a_{2}\right)^{j-i}}{(p+1+i) !(j-i) !}\right) f^{(p+1+j)}\left(a_{2}\right) \\
& +\int_{a_{1}}^{a_{2}} G_{F, n}(t, s) f^{(n)}(s) d s,
\end{aligned}
$$

where $G_{F, n}(t, s)$ is the Green's function, defined by (7).

Let $f \in C^{n}[a, b]$, and let $P_{F}$ be its 'two-point right focal' inte- 'ating $\mathrm{p} y$,ynomial for $a \leq a_{1}<a_{2} \leq b$. Then, for $n=3$ and $p=0$, (8) becomes

$$
\begin{aligned}
f(t)= & f\left(a_{1}\right)+\left(t-a_{1}\right) f^{(1)}\left(a_{2}\right)+\left(t-a_{1}\right)\left(a_{1}-a_{2}\right) f^{(2)}\left(a_{2}\right) \\
& +\int_{a_{1}}^{a_{2}} G_{1}(t, s) f^{(3)}(s) d s
\end{aligned}
$$

where

$$
G_{1}(t, s)= \begin{cases}\left(a_{1}-s\right)^{2}, & a_{1} \leq s \leq t ; \\ \left.-\left(t-a_{1}\right)\left(a_{1}-s\right)+-a_{1}\right)^{2}, & t \leq s \leq a_{2} .\end{cases}
$$

For $n=3$ and $p=1$, (8, ecomes)

$$
f(t)=f\left(a_{1}\right)+\left(t>f^{(1)}\left(a_{2}\right)+\frac{\left(t-a_{1}\right)^{2}}{2} f^{(2)}\left(a_{2}\right)+\int_{a_{1}}^{a_{2}} G_{2}(t, s) f^{(3)}(s) d s,\right.
$$

where

$$
t, s)= \begin{cases}\frac{1}{2}\left(a_{1}-s\right)^{2}+\left(t-a_{1}\right)\left(a_{1}-s\right), & a_{1} \leq s \leq t \\ -\frac{1}{2}\left(t-a_{1}\right)^{2}, & t \leq s \leq a_{2}\end{cases}
$$

The presented work is organized as follows: In Sect. 2, Levinson's inequality for 3-convex function is generalized by using two Green's functions defined by (10) and (12). In Sect. 3, Čebyšev, Grüss, and Ostrowski-type new bounds are found for the functionals involving data points of two types. In Sect. 4, the main results are applied to information theory via $f$-divergence, Rényi divergence, Rényi entropy, Shannon entropy, and Zipf-Mandelbrot law.

\section{Main results}

First we give an identity involving Jensen's difference of two different data points. Then we give an equivalent form of identity by using the Green's function defined by (10) and (12). 
Theorem 1 Let $f \in C^{3}\left[\zeta_{1}, \zeta_{2}\right]$ such that $f: I=\left[\zeta_{1}, \zeta_{2}\right] \rightarrow \mathbb{R},\left(p_{1}, \ldots, p_{n}\right) \in \mathbb{R}^{n},\left(q_{1}, \ldots, q_{m}\right) \in$ $\mathbb{R}^{m}$ such that $\sum_{\rho=1}^{n} p_{\rho}=1$ and $\sum_{\varrho=1}^{m} q_{\varrho}=1$. Also let $x_{\rho}, y_{\varrho}, \sum_{\rho=1}^{n} p_{\rho} x_{\rho}, \sum_{\varrho=1}^{m} q_{\varrho} y_{\varrho} \in I$. Then

$$
\begin{aligned}
J(f(\cdot))= & \frac{1}{2}\left[\sum_{\varrho=1}^{m} q_{\varrho} y_{\varrho}^{2}-\left(\sum_{\varrho=1}^{m} q_{\varrho} y_{\varrho}\right)^{2}-\sum_{\rho=1}^{n} p_{\rho} x_{\rho}^{2}+\left(\sum_{\rho=1}^{m} p_{\rho} x_{\rho}\right)^{2}\right] f^{(2)}\left(\zeta_{2}\right) \\
& +\int_{\zeta_{1}}^{\zeta_{2}} J\left(G_{k}(\cdot, s)\right) f^{(3)}(s) d s
\end{aligned}
$$

where

$$
J(f(\cdot))=\sum_{\varrho=1}^{m} q_{\varrho} f\left(y_{\varrho}\right)-f\left(\sum_{\varrho=1}^{m} q_{\varrho} y_{\varrho}\right)-\sum_{\rho=1}^{n} p_{\rho} f\left(x_{\rho}\right)+f\left(\sum_{\rho=1}^{n} p_{\rho} x_{\rho}\right)
$$

and

$$
\begin{aligned}
J\left(G_{k}(\cdot, s)\right)= & \sum_{\varrho=1}^{m} q_{\varrho} G_{k}\left(y_{\varrho}, s\right)-G_{k}\left(\sum_{\varrho=1}^{m} q_{\varrho} y_{\varrho}, s\right) \\
& -\sum_{\rho=1}^{n} p_{\rho} G_{k}\left(x_{\rho}, s\right)+G_{k}\left(\sum_{\rho=1}^{n} p_{\rho} x_{\rho}, s\right),
\end{aligned}
$$

for $G_{k}(\cdot, s)(k=1,2)$ defined in (10) and $(12)$ res $s_{1}$ ti ,ely.

Proof (i) For $k=1$.

Using (9) in (14), we have

$$
\begin{aligned}
J(f(\cdot))= & \sum_{\varrho=1}^{m} q_{\varrho}\left[f(1)+\left(y_{\varrho}\right)-\zeta_{1}\right) f^{(1)}\left(\zeta_{2}\right)+\left(y_{\varrho}-\zeta_{1}\right)\left(\zeta_{1}-\zeta_{2}\right) f^{(2)}\left(\zeta_{2}\right) \\
& -\left[f\left(\zeta_{1}\right)+\left(\sum_{\varrho=1}^{m} q_{\varrho} y_{\varrho}-\zeta_{1}\right) f^{(1)}\left(\zeta_{2}\right)+\left(\sum_{\varrho=1}^{m} q_{\varrho} y_{\varrho}-\zeta_{1}\right)\left(\zeta_{1}-\zeta_{2}\right) f^{(2)}\left(\zeta_{2}\right)\right. \\
& \left.+\frac{\left(\sum_{\varrho=1}^{m} q_{\varrho} y_{\varrho}-\zeta_{1}\right)^{2}}{2} f^{(2)}\left(\zeta_{2}\right)+\int_{\zeta_{1}}^{\zeta_{2}} G_{1}\left(\sum_{\varrho=1}^{m} q_{\varrho} y_{\varrho}, s\right) f^{(3)}(s) d s\right] \\
& -\sum_{\rho=1}^{n} p_{\rho}\left[f\left(\zeta_{1}\right)+\left(x_{\rho}-\zeta_{1}\right) f^{(1)}\left(\zeta_{2}\right)+\left(x_{\rho}-\zeta_{1}\right)\left(\zeta_{1}-\zeta_{2}\right) f^{(2)}\left(\zeta_{2}\right)\right. \\
& \left.+\frac{\left(x_{\rho}-\zeta_{1}\right)^{2}}{2} f^{(2)}\left(\zeta_{2}\right)+\int_{\zeta_{1}}^{\zeta_{2}} G_{1}\left(x_{\rho}, s\right) f^{(3)}(s) d s\right] \\
& +\left[f\left(\zeta_{1}\right)+\left(\sum_{\rho=1}^{n} p_{\rho} x_{\rho}-\zeta_{1}\right) f^{(1)}\left(\zeta_{2}\right)+\left(\sum_{\rho=1}^{n} p_{\rho} x_{\rho}-\zeta_{1}\right)\left(\zeta_{1}-\zeta_{2}\right) f^{(2)}\left(\zeta_{2}\right)\right. \\
& \left.+\frac{\left(\sum_{\rho=1}^{n} p_{\rho} x_{\rho}-\zeta_{1}\right)^{2}}{2} f^{(2)}\left(\zeta_{2}\right)+\int_{\zeta_{1}}^{\zeta_{2}} G_{1}\left(\sum_{\rho=1}^{n} p_{\rho} x_{\rho}, s\right) f^{(3)}(s) d s\right] .
\end{aligned}
$$




$$
\begin{aligned}
& J(f(\cdot))=f\left(\zeta_{1}\right)+\left(\sum_{\varrho=1}^{m} q_{\varrho} y_{\varrho}-\zeta_{1}\right) f^{(1)}\left(\zeta_{2}\right)+\left(\sum_{\varrho=1}^{m} q_{\varrho} y_{\varrho}-\zeta_{1}\right)\left(\zeta_{1}-\zeta_{2}\right) f^{(2)}\left(\zeta_{2}\right) \\
& +\frac{\left(\sum_{\varrho=1}^{m} q_{\varrho} y_{\varrho}^{2}-2 \zeta_{1} \sum_{\varrho=1}^{m} q_{\varrho} y_{\varrho}+\zeta_{1}^{2}\right) f^{(2)}\left(\zeta_{2}\right)}{2}+\sum_{i=1}^{m} q_{\varrho} \int_{\zeta_{1}}^{\zeta_{2}} G_{1}\left(y_{\varrho}, s\right) f^{(3)}(s) d s \\
& -f\left(\zeta_{1}\right)-\left(\sum_{\varrho=1}^{m} q_{\varrho} y_{\varrho}-\zeta_{1}\right) f^{(1)}\left(\zeta_{2}\right)-\left(\sum_{\varrho=1}^{m} q_{\varrho} y_{\varrho}-\zeta_{1}\right)\left(\zeta_{1}-\zeta_{2}\right) f^{(2)}\left(\zeta_{2}\right) \\
& -\frac{\left(\left(\sum_{\varrho=1}^{m} q_{\varrho} y_{\varrho}\right)^{2}-2 \zeta_{1} \sum_{\varrho=1}^{m} q_{\varrho} y_{\varrho}+\zeta_{1}^{2}\right) f^{(2)}\left(\zeta_{2}\right)}{2} \\
& -\int_{\zeta_{1}}^{\zeta_{2}} G_{1}\left(\sum_{\varrho=1}^{m} q_{\varrho} y_{\varrho}, s\right) f^{(3)}(s) d s \\
& -f\left(\zeta_{1}\right)-\left(\sum_{\rho=1}^{n} p_{\rho} x_{\rho}-\zeta_{1}\right) f^{(1)}\left(\zeta_{2}\right)-\left(\sum_{\rho=1}^{n} p_{\rho} x_{\rho}-\zeta_{1}\right)\left(\zeta_{1}-\zeta_{2} \cdot(2)\left(\zeta_{2}\right)\right. \\
& -\frac{\left(\sum_{\rho=1}^{n} p_{\rho} x_{\rho}^{2}-2 \zeta_{1} \sum_{\rho=1}^{n} p_{\rho} x_{\rho}+\zeta_{1}^{2}\right) f^{(2)}\left(\zeta_{2}\right)}{2}-\sum_{\rho=1} v_{1}\left(x_{\rho}, s\right) f^{(3)}(s) d s \\
& +f\left(\zeta_{1}\right)+\left(\sum_{\rho=1}^{n} p_{\rho} x_{\rho}-\zeta_{1}\right) f^{(1)}\left(\zeta_{2}\right)+\left(\sum_{n=1}^{n} p_{\rho} x_{1}-\zeta_{1}\right)\left(\zeta_{1}-\zeta_{2}\right) f^{(2)}\left(\zeta_{2}\right) \\
& \left.+\frac{\left(\left(\sum_{\rho=1}^{n} p_{\rho} x_{\rho}\right)^{2}-2 \zeta_{1} \sum_{\rho=1}^{n} p_{\rho}\right.}{2}+\zeta_{1}\right)^{(2)}\left(\zeta_{2}\right) \\
& +\int_{\zeta_{1}}^{\zeta_{2}} G_{1}\left(\sum_{\rho=1}^{n} p_{\rho}, s\right) f \\
& J(f(\cdot))=\frac{1}{2}\left[\sum_{\varrho=1}^{m} q v_{\varrho}^{2}-\left(\sum_{\varrho=1}^{m} q_{\varrho} y_{\varrho}\right)^{2}-\sum_{\rho=1}^{n} p_{\rho} x_{\rho}^{2}+\left(\sum_{\rho=1}^{n} p_{\rho} x_{\rho}\right)^{2}\right] f^{(2)}\left(\zeta_{2}\right) \\
& +\sum_{\varrho=}^{\zeta_{2}} G_{1}\left(y_{\varrho}, s\right) f^{(3)}(s) d s-\int_{\zeta_{1}}^{\zeta_{2}} G_{1}\left(\sum_{\varrho=1}^{m} q_{\varrho} y_{\varrho}, s\right) f^{(3)}(s) d s \\
& -\sum_{\rho=1}^{n} p_{\rho} \int_{\zeta_{1}}^{\zeta_{2}} G_{1}\left(x_{\rho}, s\right) f^{(3)}(s) d s+\int_{\zeta_{1}}^{\zeta_{2}} G_{1}\left(\sum_{\rho=1}^{n} p_{\rho} x_{\rho}, s\right) f^{(3)}(s) d s
\end{aligned}
$$

After rearranging, we have (13).

(ii) For $k=2$

Using (11) in (14) and following similar steps as in the proof of (i), we get (13).

Corollary 1 Let $f \in C^{3}[0,2 \alpha]$ such that $f: I=[0,2 \alpha] \rightarrow \mathbb{R}, x_{1}, \ldots, x_{n} \in(0, \alpha),\left(p_{1}, \ldots, p_{n}\right) \in$ $\mathbb{R}^{n}$ such that $\sum_{\rho=1}^{n} p_{\rho}=1$. Also let $x_{\rho}, \sum_{\rho=1}^{n} p_{\rho}\left(2 \alpha-x_{\rho}\right), \sum_{\rho=1}^{n} p_{\rho} x_{\rho} \in I$. Then

$$
J(f(\cdot))=\int_{\zeta_{1}}^{\zeta_{2}} J\left(G_{k}(\cdot, s)\right) f^{(3)}(s) d s, \quad 0 \leq \zeta_{1}<\zeta_{2} \leq 2 \alpha,
$$

where $J(f(\cdot))$ and $J(G(\cdot, s))$ are defined in (14) and (15) respectively. 
Proof Choosing $I=[0,2 \alpha], y_{\varrho}=\left(2 \alpha-x_{\rho}\right), x_{1}, \ldots, x_{n} \in(0, \alpha), p_{\rho}=q_{\varrho}$, and $m=n$ in Theorem 1 , after simplification we get (16).

Theorem 2 Let $f: I=\left[\zeta_{1}, \zeta_{2}\right] \rightarrow \mathbb{R}$ be a 3-convex function. Also let $\left(p_{1}, \ldots, p_{n}\right) \in \mathbb{R}^{n}$, $\left(q_{1}, \ldots, q_{m}\right) \in \mathbb{R}^{m}$ be such that $\sum_{\rho=1}^{n} p_{\rho}=1$ and $\sum_{\varrho=1}^{m} q_{\varrho}=1$ and $x_{\rho}, y_{\varrho}, \sum_{\rho=1}^{n} p_{\rho} x_{\rho}$, $\sum_{\varrho=1}^{m} q_{\varrho} y_{\varrho} \in I$.

If

$$
\left[\sum_{\varrho=1}^{m} q_{\varrho} y_{\varrho}^{2}-\left(\sum_{\varrho=1}^{m} q_{\varrho} y_{\varrho}\right)^{2}-\sum_{\rho=1}^{n} p_{\rho} x_{\rho}^{2}+\left(\sum_{\rho=1}^{n} p_{\rho} x_{\rho}\right)^{2}\right] f^{(2)}\left(\zeta_{2}\right) \geq 0
$$

then the following statements are equivalent:

For $f \in C^{3}\left[\zeta_{1}, \zeta_{2}\right]$,

$$
\sum_{\rho=1}^{n} p_{\rho} f\left(x_{\rho}\right)-f\left(\sum_{\rho=1}^{n} p_{\rho} x_{\rho}\right) \leq \sum_{\varrho=1}^{m} q_{\varrho} f\left(y_{\varrho}\right)-f\left(\sum_{\varrho=1}^{m} q_{\varrho} y_{\varrho}\right)
$$

For all $s \in I$,

$$
\left.\sum_{\rho=1}^{n} p_{\rho} G_{k}\left(x_{\rho}, s\right)-G_{k}\left(\sum_{\rho=1}^{n} p_{\rho} x_{\rho}, s\right) \leq \sum_{\varrho=1}^{m} 1_{\varrho}, s\right)-G_{k}\left(\sum_{\varrho=1}^{m} q_{\varrho} y_{\varrho}, s\right)
$$

where $G_{k}(\cdot, s)$ are defined by (10) an 12, for $k=1,2$ respectively.

Moreover, inequality in (18) is evers if ir equality in (19) is reversed.

Proof $(18) \Rightarrow(19)$ : Let (18) be valic Then, as the function $G_{k}(\cdot, s)(s \in I)$ is also continuous and 3-convex, it follow, that also for this function (18) holds, i.e., (19) is valid.

$(19) \Rightarrow(18)$ : If $f$ is $\mathbf{3}$ onvex, hen without loss of generality we can suppose that there exists the third d rivative $u_{1}$. Let $f \in C^{3}\left[\zeta_{1}, \zeta_{2}\right]$ be a 3 -convex function and (19) hold. Then we can represent fur $1 f$ in the form (9). Now, by means of some simple calculations, we can $y$.

$$
\begin{aligned}
\left.\sum_{=1}^{m} q_{\varrho} \jmath_{\varrho}\right)-f\left(\sum_{\varrho=1}^{m} q_{\varrho} y_{\varrho}\right)-\sum_{\rho=1}^{n} p_{\rho} f\left(x_{\rho}\right)+f\left(\sum_{\rho=1}^{n} p_{\rho} x_{\rho}\right) \\
=\frac{1}{2}\left[\sum_{\varrho=1}^{m} q_{\varrho} y_{\varrho}^{2}-\left(\sum_{\varrho=1}^{m} q_{\varrho} y_{\varrho}\right)^{2}-\sum_{\rho=1}^{n} p_{\rho} x_{\rho}^{2}+\left(\sum_{\rho=1}^{n} p_{\rho} x_{\rho}\right)^{2}\right] f^{(2)}\left(\zeta_{2}\right) \\
+\int_{\zeta_{1}}^{\zeta_{2}}\left(\sum_{\varrho=1}^{m} q_{\varrho} G_{k}\left(y_{\varrho}, s\right)-G_{k}\left(\sum_{\varrho=1}^{m} q_{\varrho}\left(y_{\varrho}, s\right)\right)\right. \\
\left.\quad-\sum_{\rho=1}^{n} p_{\rho} G_{k}\left(x_{\rho}, s\right)+G_{k}\left(\sum_{\rho=1}^{n} p_{\rho} x_{\rho}, s\right)\right) f^{(3)}(s) d s .
\end{aligned}
$$

By the convexity of $f$, we have $f^{(3)}(s) \geq 0$ for all $s \in I$. Hence, if for every $s \in I$, (19) is valid, then it follows that for every 3-convex function $f: I \rightarrow \mathbb{R}$, with $f \in C^{3}\left[\zeta_{1}, \zeta_{2}\right]$, (18) is valid. 
Remark 1 If the expression

$$
\sum_{\varrho=1}^{m} q_{\varrho} y_{\varrho}^{2}-\left(\sum_{\varrho=1}^{m} q_{\varrho} y_{\varrho}\right)^{2}-\sum_{\rho=1}^{n} p_{\rho} x_{\rho}^{2}+\left(\sum_{\rho=1}^{n} p_{\rho} x_{\rho}\right)^{2}
$$

and $f^{(2)}\left(\zeta_{2}\right)$ have different signs in (17), then inequalities (18) and (19) are reversed.

Next we have the results about generalization of Bullen-type inequality (for real weightc given in [2] (see also [16] and [11]).

Corollary 2 Let $f: I=\left[\zeta_{1}, \zeta_{2}\right] \rightarrow \mathbb{R}$ be a 3-convex function and $f \in C^{3}\left[\zeta_{1}, \zeta_{2}\right], \lambda \ldots, x_{n}$, $y_{1}, \ldots, y_{m} \in I$ such that

$$
\max \left\{x_{1}, \ldots, x_{n}\right\} \leq \min \left\{y_{1}, \ldots, y_{m}\right\}
$$

and

$$
x_{1}+y_{1}=\cdots=x_{n}+y_{m}
$$

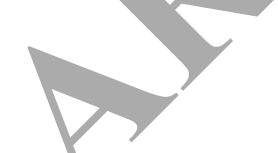

Also let $\left(p_{1}, \ldots, p_{n}\right) \in \mathbb{R}^{n},\left(q_{1}, \ldots, q_{m}\right) \in \mathbb{R}^{m}$ be swat $\sum_{p=1}^{\prime} p_{\rho}=1$ and $\sum_{\varrho=1}^{m} q_{\varrho}=1$ and $x_{\rho}, y_{\varrho}, \sum_{\rho=1}^{n} p_{\rho} x_{\rho}, \sum_{\varrho=1}^{m} q_{\varrho} y_{\varrho} \in I$.If(17) hol then 8) and (19) are equivalent.

Proof By choosing $x_{\rho}$ and $y_{\varrho}$ such th condition. 20) and (21) hold in Theorem 2, we get the required result.

Remark 2 If $p_{\rho}=q_{\varrho}$ are pistivive a ' $x_{\rho}, y_{\varrho}$ satisfy (20) and (21), then inequality (18) reduces to Bullen's inequ lity given in 116, p. 32, Theorem 2] for $m=n$.

Next we have a gener. form (for real weights) of Bullen-type inequality given in [17] (see also [16,

Corol vy 3 Letf: $Y=\left[\zeta_{1}, \zeta_{2}\right] \rightarrow \mathbb{R}$ be a 3-convex function and $f \in C^{3}\left[\zeta_{1}, \zeta_{2}\right],\left(p_{1}, \ldots, p_{n}\right) \in$ $\mathbb{R}^{n},\left(q_{1}, . q_{m}\right) \in \mathbb{R}^{m}$ be such that $\sum_{\rho=1}^{n} p_{\rho}=1$ and $\sum_{\varrho=1}^{m} q_{\varrho}=1$. Also let $x_{1}, \ldots, x_{n}$ a. $y_{1}, \ldots, y_{m} \in I$ be such that $x_{\rho}+y_{\varrho}=2 c$, and for $\rho=1, \ldots, n, x_{\rho}+x_{n-\rho+1}$ and $\frac{p_{\rho} x_{\rho}}{p_{\rho}}-\frac{\rho+1}{1-\rho+1} \leq c$.If (17) holds, then (18) and (19) are equivalent.

P) jof Using Theorem 2 with the conditions given in the statement, we get the required result.

Remark 3 In Theorem 2, if $m=n, p_{\rho}=q_{\varrho}$ are positive, $x_{\rho}+y_{\varrho}=2 c, x_{\rho}+x_{n-\rho+1}$ and $\frac{p_{\rho} x_{\rho}+p_{n-\rho+1} x_{n-\rho+1}}{p_{\rho}+p_{n-\rho+1}} \leq c$. Then (18) reduces to a generalized form of Bullen's inequality defined in $[16$, p. 32, Theorem 4].

In [15], Mercer made a notable work by replacing condition (21) of symmetric distribution of points $x_{\rho}$ and $y_{\varrho}$ with symmetric variances of points $x_{\rho}$ and $y_{\varrho}$ for $\rho=1, \ldots, n$ and $\varrho=1, \ldots, m$.

So in the next result we use Mercer's condition (6), but for $\rho=\varrho$ and $m=n$. 
Corollary 4 Let $f: I=\left[\zeta_{1}, \zeta_{1}\right] \rightarrow \mathbb{R}$ be a 3-convex function and $f \in C^{3}\left[\zeta_{1}, \zeta_{2}\right], p_{\rho}, q_{\rho}$ be positive such that $\sum_{\rho=1}^{n} p_{\rho}=1$ and $\sum_{\rho=1}^{n} q_{\rho}=1$. Also let $x_{\rho}, y_{\rho}$ satisfy $(20)$ and

$$
\sum_{\rho=1}^{n} p_{\rho}\left(x_{\rho}-\sum_{\rho=1}^{n} p_{\rho} x_{\rho}\right)^{2}=\sum_{\rho=1}^{n} p_{\rho}\left(y_{\rho}-\sum_{\rho=1}^{n} q_{\rho} y_{\rho}\right)^{2} .
$$

If (17) holds, then (18) and (19) are equivalent.

Proof For positive weights, using (6) and (20) in Theorem 2, we get the required recult.

Next we have the results that lean on the generalization of Levinson-ty $/ \mathrm{e}$ in rality given in [12] (see also [16]).

Corollary 5 Let $f: I=[0,2 \alpha] \rightarrow \mathbb{R}$ be a 3-convex function and $f \in \mathcal{E}^{3}\left[0, \ldots, x_{1}, \ldots, x_{n} \in\right.$ $(0, \alpha),\left(p_{1}, \ldots, p_{n}\right) \in \mathbb{R}^{n}$ and $\sum_{\rho=1}^{n} p_{\rho}=1$. Also let $x_{\rho}, \sum_{\rho=1}^{n} p_{\rho}\left(2--x_{\rho} \sum_{\rho=1}^{n} p_{\rho} x_{\rho} \in I\right.$. Then the following are equivalent:

$$
\sum_{\rho=1}^{n} p_{\rho} f\left(x_{\rho}\right)-f\left(\sum_{\rho=1}^{n} p_{\rho} x_{\rho}\right) \leq \sum_{\rho=1}^{n} p_{\rho} f\left(2 \alpha-x_{\rho}\right)-f\left(\sum_{\rho=1}^{n} p_{\rho}\left(2 \alpha-x_{\rho}\right)\right) .
$$

For all $s \in I$,

$$
\begin{array}{r}
\sum_{\rho=1}^{n} p_{\rho} G_{k}\left(x_{\rho}, s\right)-G_{k}\left(\sum_{\rho}^{n} r \hat{x_{\rho}, s}\right) \leq \sum_{\rho=1}^{n} p_{\rho} G_{k}\left(2 \alpha-x_{\rho}, s\right) \\
-G_{k}\left(\sum_{\rho=1}^{n} p_{\rho}\left(2 \alpha-x_{\rho}\right), s\right),
\end{array}
$$

where $G_{k}(\cdot, s)$ is a jur

(10) and (12) for $k=1,2$ respectively.

Proof $I=n_{n 1},\left(x_{1}, \ldots, x_{n}\right) \in(0, \alpha), p_{\rho}=q_{\varrho}, m=n$, and $y_{\varrho}=\left(2 \alpha-x_{\rho}\right)$ in Theorem 2 with $0 \leq s, \zeta_{2} \leq 2 \alpha$, we get the required result.

Rema. 4 In Corollary 5, if $p_{\rho}$ are positive, then inequality (23) reduces to Levinson's in quality given in [16, p. 32, Theorem 1].

\section{New bounds for Levinson-type functionals}

Consider the Čebyšev functional for two Lebesgue integrable functions $f_{1}, f_{2}:\left[\zeta_{1}, \zeta_{2}\right] \rightarrow \mathbb{R}$

$$
\begin{aligned}
\Theta\left(f_{1}, f_{2}\right)= & \frac{1}{\zeta_{2}-\zeta_{1}} \int_{\zeta_{1}}^{\zeta_{2}} f_{1}(x) f_{2}(x) d x \\
& -\frac{1}{\zeta_{2}-\zeta_{1}} \int_{\zeta_{1}}^{\zeta_{2}} f_{1}(x) d x \times \frac{1}{\zeta_{2}-\zeta_{1}} \int_{\zeta_{1}}^{\zeta_{2}} f_{2}(x) d x,
\end{aligned}
$$

where the integrals are assumed to exist. 
Theorem $\mathbf{F}([3])$ Let $f_{1}:\left[\zeta_{1}, \zeta_{2}\right] \rightarrow \mathbb{R}$ be a Lebesgue integrable function and $f_{2}:\left[\zeta_{1}, \zeta_{2}\right] \rightarrow \mathbb{R}$ be an absolutely continuous function with $\left(\cdot,-\zeta_{1}\right)\left(\cdot,-\zeta_{2}\right)\left[f_{2}^{\prime}\right]^{2} \in L\left[\zeta_{1}, \zeta_{2}\right]$. Then

$$
\left|\Theta\left(f_{1}, f_{2}\right)\right| \leq \frac{1}{\sqrt{2}}\left[\Theta\left(f_{1}, f_{1}\right)\right]^{\frac{1}{2}} \frac{1}{\sqrt{\zeta_{2}-\zeta_{1}}}\left(\int_{\zeta_{1}}^{\zeta_{2}}\left(t-\zeta_{1}\right)\left(\zeta_{2}-t\right)\left[f_{2}^{\prime}(t)\right]^{2} d t\right)^{\frac{1}{2}} .
$$

$\frac{1}{\sqrt{2}}$ is the best possible.

Theorem G ([3]) Let $f_{1}:\left[\zeta_{1}, \zeta_{2}\right] \rightarrow \mathbb{R}$ be absolutely continuous with $f_{1}^{\prime} \in L_{\infty}\left[\zeta_{1}, \zeta_{2}\right]$, ard let $f_{2}:\left[\zeta_{1}, \zeta_{2}\right] \rightarrow \mathbb{R}$ be monotonic nondecreasing on $\left[\zeta_{1}, \zeta_{2}\right]$. Then

$$
\left|\Theta\left(f_{1}, f_{2}\right)\right| \leq \frac{1}{2\left(\zeta_{2}-\zeta_{1}\right)}\left\|f^{\prime}\right\|_{\infty} \int_{\zeta_{1}}^{\zeta_{2}}\left(t-\zeta_{1}\right)\left(\zeta_{2}-t\right)\left[f_{2}^{\prime}(t)\right] d f_{2}(t)
$$

$\frac{1}{2}$ is the best possible.

In the next result we construct the Čebyšev-type bo nd for ou anctional defined in (5).

Theorem 3 Let $f \in C^{3}\left[\zeta_{1}, \zeta_{2}\right]$ be such that $\left.f .1=\zeta_{1}, \zeta_{2}\right] \rightarrow \mathbb{R}$ and $f^{(3)}(\cdot)$ is absolutely continuous with $\left(\cdot-\zeta_{1}\right)\left(\zeta_{2}-\cdot\right)\left[f^{(4)}\right]^{2} \in L\left[\zeta_{1}, \zeta_{2}\right]$. olet $\left.p_{1}, \ldots, p_{n}\right) \in \mathbb{R}^{n},\left(q_{1}, \ldots, q_{m}\right) \in \mathbb{R}^{m}$ be such that $\sum_{\rho=1}^{n} p_{\rho}=1, \sum_{\varrho=1}^{m} q_{\varrho}=1, x, y_{\xi}, \sum_{R=1}^{n}, x_{\rho}, \sum_{\varrho=1}^{m} q_{\varrho} y_{\varrho} \in I$. Then

$$
\begin{aligned}
& J(f(\cdot))=\frac{1}{2}\left[\sum_{\varrho=1}^{m} q_{\varrho} y_{\varrho}^{2}-\left(\sum_{\varrho=1}\right)^{2}-\sum_{\rho=1}^{n} p_{\rho} x_{\rho}^{2}+\left(\sum_{\varrho=1}^{m} p_{\rho} x_{\rho}\right)^{2}\right] f^{(2)}\left(\zeta_{2}\right) \\
& +\frac{f^{(2)}\left(\zeta_{2}\right.}{\left(\zeta_{2}\right.}-f^{(2)}\left(\zeta_{1}\right) \int_{\zeta_{1}}^{\zeta_{2}} J\left(G_{k}(\cdot, s)\right) f^{(3)}(s) d s+\mathcal{R}_{3}\left(\zeta_{1}, \zeta_{2} ; f\right),
\end{aligned}
$$

where $J(f \rightarrow J(G,(\cdot)$,$) are defined in (14) and (15) respectively, and the remainder$ $\mathcal{R}_{3}\left(\zeta_{2}, f\right)$ aticfie, the bound

$$
\begin{aligned}
\mathcal{R}_{3}\left(\zeta_{1}, \zeta_{2} ; f\right) \mid \leq & \frac{\zeta_{2}-\zeta_{1}}{\sqrt{2}}\left[\Theta\left(J\left(G_{k}(\cdot, s)\right), J\left(G_{k}(\cdot, s)\right)\right)\right]^{\frac{1}{2}} \times \\
& \frac{1}{\sqrt{\zeta_{2}-\zeta_{1}}}\left(\int_{\zeta_{1}}^{\zeta_{2}}\left(s-\zeta_{1}\right)\left(\zeta_{2}-s\right)\left[f^{(4)}(s)\right] d s\right)^{\frac{1}{2}}
\end{aligned}
$$

for $G_{k}(\cdot, s)(k=1,2)$ defined in (10) and (12) respectively.

Proof Setting $f_{1} \mapsto J\left(G_{k}(\cdot, s)\right)$ and $f_{2} \mapsto f^{(3)}$ in Theorem F, we get

$$
\begin{gathered}
\left|\frac{1}{\zeta_{2}-\zeta_{1}} \int_{\zeta_{1}}^{\zeta_{2}} J\left(G_{k}(\cdot, s)\right) f^{(3)}(s) d s-\frac{1}{\zeta_{2}-\zeta_{1}} \int_{\zeta_{1}}^{\zeta_{2}} J\left(G_{k}(\cdot, s)\right) d s \times \frac{1}{\zeta_{2}-\zeta_{1}} \int_{\zeta_{1}}^{\zeta_{2}} f^{(3)}(s) d s\right| \\
\leq \frac{1}{\sqrt{2}}\left[\Theta\left(J\left(G_{k}(\cdot, s)\right), J\left(G_{k}(\cdot, s)\right)\right)\right]^{\frac{1}{2}} \frac{1}{\sqrt{\zeta_{2}-\zeta_{1}}}\left(\int_{\zeta_{1}}^{\zeta_{2}}\left(s-\zeta_{1}\right)\left(\zeta_{2}-s\right)\left[f^{(4)}(s)\right] d s\right)^{\frac{1}{2}},
\end{gathered}
$$




$$
\begin{aligned}
& \left|\frac{1}{\zeta_{2}-\zeta_{1}} \int_{\zeta_{1}}^{\zeta_{2}} J\left(G_{k}(\cdot, s)\right) f^{(3)}(s) d s-\frac{f^{(2)}\left(\zeta_{2}\right)-f^{(2)}\left(\zeta_{1}\right)}{\left(\zeta_{2}-\zeta_{1}\right)^{2}} \int_{\zeta_{1}}^{\zeta_{2}} J\left(G_{k}(\cdot, s)\right) d s\right| \\
& \quad \leq \frac{1}{\sqrt{2}}\left[\Theta\left(J\left(G_{k}(\cdot, s)\right), J\left(G_{k}(\cdot, s)\right)\right)\right]^{\frac{1}{2}} \frac{1}{\sqrt{\zeta_{2}-\zeta_{1}}}\left(\int_{\zeta_{1}}^{\zeta_{2}}\left(s-\zeta_{1}\right)\left(\zeta_{2}-s\right)\left[f^{(4)}(s)\right] d s\right)^{\frac{1}{2}} .
\end{aligned}
$$

Multiplying $\left(\zeta_{2}-\zeta_{1}\right)$ on both sides of the above inequality and using estimation (29), we get

$$
\int_{\zeta_{1}}^{\zeta_{2}} J\left(G_{k}(\cdot, s)\right) f^{(3)} d s=\frac{f^{(2)}\left(\zeta_{2}\right)-f^{(2)}\left(\zeta_{1}\right)}{\left(\zeta_{2}-\zeta_{1}\right)} \int_{\zeta_{1}}^{\zeta_{2}} J\left(G_{k}(\cdot, s)\right) d s+\mathcal{R}_{3}\left(\zeta_{1}, \zeta_{1} ; f\right)
$$

Using identity (13), we get (28).

In the next result the bounds of Grüss-type inequalities are estim tea

Theorem 4 Let $f \in C^{3}\left[\zeta_{1}, \zeta_{2}\right]$ be such that $f: I=\left[\zeta_{1}, \zeta_{2}\right] \rightarrow \mathbb{R}_{(3)}$ (3) (.) ahsolutely continuous and $f^{(4)}(\cdot) \geq 0$ a.e. on $\left[\zeta_{1}, \zeta_{2}\right]$. Also let $\left(p_{1}, \ldots, p_{n}\right) \in \mathbb{R}^{n},\left(q_{1}, . . \tau_{m}\right) \in \mathbb{R}^{m}$ be such that $\sum_{\rho=1}^{n} p_{\rho}=1, \sum_{\varrho=1}^{m} q_{\varrho}=1, x_{\rho}, y_{\varrho}, \sum_{\rho=1}^{n} p_{\rho} x_{\rho}, \sum_{\varrho=1}^{m} q_{\varrho} y_{\varrho} \in$.ron identity (28) holds, where the remainder satisfies the estimation

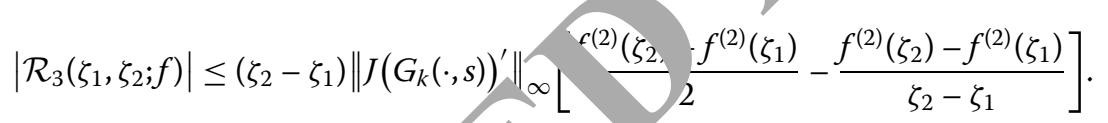

Proof Setting $f_{1} \mapsto J\left(G_{k}(\cdot, s)\right)$ an $\sqrt{2}_{2} \mapsto$, in 1 heorem G, we get

$$
\begin{aligned}
& \left.\mid \frac{1}{\zeta_{2}-\zeta_{1}} \int_{\zeta_{1}}^{\zeta_{2}} J\left(G_{k}(, s)\right)\right) f^{(3)}(s) d s-\frac{1}{\zeta_{2}-\zeta_{1}} \int_{\zeta_{1}}^{\zeta_{2}} J\left(G_{k}(\cdot, s)\right) d s \cdot \frac{1}{\zeta_{2}-\zeta_{1}} \int_{\zeta_{1}}^{\zeta_{2}} f^{(3)}(s) d s \mid \\
& \quad \leq \frac{1}{2}\left\|J\left(G^{-}(\cdot s)\right)^{\prime}\right\|_{\infty} \frac{1}{\zeta_{2}-\zeta_{1}} \int_{\zeta_{1}}^{\zeta_{2}}\left(s-\zeta_{1}\right)\left(\zeta_{2}-s\right)\left[f^{(4)}(s)\right] d s .
\end{aligned}
$$

Since

$$
\begin{aligned}
& \int_{\zeta_{2}}^{\zeta_{2}}\left(s=\zeta_{1}\right)\left(\zeta_{2}-s\right)\left[f^{(4)}(s)\right] d s \\
& =\int_{\zeta_{1}}^{\zeta_{2}}\left[2 s-\zeta_{1}-\zeta_{2}\right] f^{3}(s) d s \\
& =\left(\zeta_{2}-\zeta_{1}\right)\left[f^{(2)}\left(\zeta_{2}\right)+f^{(2)}\left(\zeta_{1}\right)\right]-2\left(f^{(2)}\left(\zeta_{2}\right)-f^{(2)}\left(\zeta_{1}\right)\right),
\end{aligned}
$$

using (13), (31), and (32), we have (28).

Ostrowski-type bounds for a newly constructed functional defined in (5).

Theorem 5 Let $f \in C^{3}\left[\zeta_{1}, \zeta_{2}\right]$ be such that $f: I=\left[\zeta_{1}, \zeta_{2}\right] \rightarrow \mathbb{R}$ and $f^{(2)}(\cdot)$ is absolutely continuous. Also let $\left(p_{1}, \ldots, p_{n}\right) \in \mathbb{R}^{n},\left(q_{1}, \ldots, q_{m}\right) \in \mathbb{R}^{m}$ be such that $\sum_{\rho=1}^{n} p_{\rho}=1, \sum_{\varrho=1}^{m} q_{\varrho}=1$, $x_{\rho}, y_{\varrho}, \sum_{\rho=1}^{n} p_{\rho} x_{\rho}, \sum_{\varrho=1}^{m} q_{\varrho} y_{\varrho} \in I$. Also let $(r, s)$ be a pair of conjugate exponents, that is, 


$$
\begin{aligned}
& 1 \leq r, s, \leq \infty, \frac{1}{r}+\frac{1}{s}=1 \text {. If }\left|f^{(3)}\right|^{r}:\left[\zeta_{1}, \zeta_{2}\right] \rightarrow \mathbb{R} \text { is a Riemann integrable function, then } \\
& \left|J(f(\cdot))-\frac{1}{2}\left[\sum_{\varrho=1}^{m} q_{\varrho} y_{\varrho}^{2}-\left(\sum_{\varrho=1}^{m} q_{\varrho} y_{\varrho}\right)^{2}-\sum_{\rho=1}^{n} p_{\rho} x_{\rho}^{2}+\left(\sum_{\varrho=1}^{m} p_{\rho} x_{\rho}\right)^{2}\right] f^{(2)}\left(\zeta_{2}\right)\right| \\
& \quad \leq\left\|f^{(3)}\right\|_{r}\left(\int_{\zeta_{1}}^{\zeta_{2}}\left|J\left(G_{k}(\cdot, s)\right) d s\right|^{s}\right)^{\frac{1}{s}} .
\end{aligned}
$$

Proof Rearrange identity (13) in the following way:

$$
\begin{aligned}
& \mid J(f(\cdot))-\frac{1}{2}\left(\sum_{\varrho=1}^{m} q_{\varrho} y_{\varrho}^{2}-\left(\sum_{\varrho=1}^{m} q_{\varrho} y_{\varrho}\right)^{2}-\sum_{\rho=1}^{n} p_{\rho} x_{\rho}^{2}+\left(\sum_{\rho=1}^{m} p_{\rho} x_{\rho}\right)^{2}\right) f^{(2)}\left(\zeta_{2}\right) \\
& \quad \leq \int_{\zeta_{1}}^{\zeta_{2}} J\left(G_{k}(\cdot, s)\right) f^{(3)}(s) d s .
\end{aligned}
$$

Employing the classical Holder's inequality to R.H.S of (34) yiel "33).

\section{Application to information theory}

The idea of Shannon entropy is the focal job of hypoth esis once in a while alluded as measure of uncertainty. The entropy of a $/$ dom riable is characterized regarding its probability distribution and can be shown to decent measure of randomness or uncertainty. Shannon entropy permits vv 'uate the normal least number of bits expected to encode a series of images de endent the letters in order size and the recurrence of the symbols.

Divergences between probability stributions have been acquainted with measure of the difference betwee them. A variety of sorts of divergences exist, for instance the $f$ difference (particularly, $11 \mathrm{~h}-\mathrm{ck}$-Leibler divergence, Hellinger distance, and total variation distance), $R_{c}$. Mivergence, Jensen-Shannon divergence, and so forth (see [13, 21]). There are lot of papers managing inequalities and entropies, see, e.g., [8, 10, 20] and the ref enc s ther $-1 n$. Jensen's inequality assumes a crucial role in a portion of these inecuaritie $\eta$ any case, Jensen's inequality deals with one sort of information focuses and L. nson's is,equality manages two types of information points.

$\mathrm{Zip}$ law is one of the central laws in data science, and it has been utilized in linguistics. George Zipf in 1932 found that we can tally how frequently each word shows up in the content. So on the off chance that we rank (r) word as per the recurrence of word event $(f)$, at that point the result of these two numbers is steady $(C): C=r \times f$. Aside from the utilization of this law in data science and linguistics, Zipf's law is utilized in city population, sun powered flare power, site traffic, earthquake magnitude, the span of moon pits, and so forth. In financial aspects this distribution is known as the Pareto law, which analyzes the distribution of the wealthiest individuals in the community [6, p. 125]. These two laws are equivalent in the mathematical sense, yet they are involved in different contexts [7, p. 294].

\subsection{Csiszár divergence}

In $[4,5]$ Csiszár gave the following definition: 
Definition 1 Let $f$ be a convex function from $\mathbb{R}^{+}$to $\mathbb{R}^{+}$. Let $\tilde{\mathbf{r}}, \tilde{\mathbf{k}} \in \mathbb{R}_{+}^{n}$ be such that $\sum_{s=1}^{n} r_{s}=$ 1 and $\sum_{s=1}^{n} q_{s}=1$. Then an $f$-divergence functional is defined by

$$
I_{f}(\tilde{\mathbf{r}}, \tilde{\mathbf{k}}):=\sum_{s=1}^{n} q_{s} f\left(\frac{r_{s}}{q_{s}}\right) .
$$

By defining the following:

$$
f(0):=\lim _{x \rightarrow 0^{+}} f(x) ; \quad 0 f\left(\frac{0}{0}\right):=0 ; \quad 0 f\left(\frac{a}{0}\right):=\lim _{x \rightarrow 0^{+}} x f\left(\frac{a}{0}\right), \quad a>0,
$$

he stated that nonnegative probability distributions can also be used.

Using the definition of $f$-divergence functional, Horv́ath et al. [9] gave functional:

Definition 2 Let $I$ be an interval contained in $\mathbb{R}$ and $f: I \rightarrow \mathbb{R}$ be a ful. in. Also let $\tilde{\mathbf{r}}=\left(r_{1}, \ldots, r_{n}\right) \in \mathbb{R}^{n}$ and $\tilde{\mathbf{k}}=\left(k_{1}, \ldots, k_{n}\right) \in(0, \infty)^{n}$ be such that

$$
\frac{r_{s}}{k_{s}} \in I, \quad s=1, \ldots, n \text {. }
$$

Then

$$
\hat{I}_{f}(\tilde{\mathbf{r}}, \tilde{\mathbf{k}}):=\sum_{s=1}^{n} k_{s} f\left(\frac{r_{s}}{k_{s}}\right)
$$

We apply a generalized form if Bulleı no quality (18) (for positive weights) to $\hat{I}_{f}(\tilde{\mathbf{r}}, \tilde{\mathbf{k}})$.

Let us denote the followin, se. ' assumptions by $\mathcal{G}$ :

Let $f: I=[\alpha, \beta] \rightarrow \mathbb{R}$ be a 3-convt anction. Also let $\left(p_{1}, \ldots, p_{n}\right) \in \mathbb{R}^{+},\left(q_{1}, \ldots, q_{m}\right) \in \mathbb{R}^{+}$ be such that $\sum_{s=1}^{n} p_{s}=$ and $\sum_{s=1}^{m} q_{s}=1$ and $x_{s}, y_{s}, \sum_{s=1}^{n} p_{s} x_{s}, \sum_{s=1}^{m} q_{s} y_{s} \in I$.

\section{Theorem 6 Ass $\mathcal{G}$.}

Let $\left.\tilde{\mathbf{r}}=\left(r_{1}, \ldots, r_{n}\right), \ldots, k_{1}, \ldots, k_{n}\right)$ be in $(0, \infty)^{n}$, and $\tilde{\mathbf{w}}=\left(w_{1}, \ldots, w_{m}\right), \tilde{\mathbf{t}}=\left(t_{1}, \ldots, t_{m}\right)$ be in $(0, \infty)^{m}$ suc that

$$
\frac{r_{s}}{k_{s}} \in, \quad s=1, \ldots, n,
$$

and

$$
\frac{w_{u}}{t_{u}} \in I, \quad u=1, \ldots, m
$$

Then

$$
\text { (i) } \frac{1}{\sum_{s=1}^{n} k_{s}} \hat{I}_{f}(\tilde{\mathbf{r}}, \tilde{\mathbf{k}})-f\left(\sum_{s=1}^{n} \frac{r_{s}}{\sum_{s=1}^{n} k_{s}}\right) \leq \frac{1}{\sum_{u=1}^{m} t_{u}} \hat{I}_{f}(\tilde{\mathbf{w}}, \tilde{\mathbf{t}})-f\left(\sum_{u=1}^{m} \frac{w_{u}}{\sum_{u=1}^{m} t_{u}}\right) .
$$

(ii) If $x \rightarrow x f(x)(x \in[a, b])$ is 3-convex, then

$$
\frac{1}{\sum_{s=1}^{n} k_{s}} \hat{I}_{d d f}(\tilde{\mathbf{r}}, \tilde{\mathbf{k}})-f\left(\sum_{s=1}^{n} \frac{r_{s}}{\sum_{s=1}^{n} k_{s}}\right) \leq \frac{1}{\sum_{u=1}^{m} t_{u}} \hat{I}_{d d f}(\tilde{\mathbf{w}}, \tilde{\mathbf{t}})-f\left(\sum_{u=1}^{m} \frac{w_{u}}{\sum_{u=1}^{m} t_{u}}\right),
$$


where

$$
\hat{I}_{i d f}(\tilde{\mathbf{r}}, \tilde{\mathbf{k}})=\sum_{s=1}^{n} r_{s} f\left(\frac{r_{s}}{k_{s}}\right)
$$

and

$$
\hat{I}_{i d f}(\tilde{\mathbf{w}}, \tilde{\mathbf{t}})=\sum_{u=1}^{m} w_{u} f\left(\frac{w_{u}}{t_{u}}\right)
$$

Proof (i) Taking $p_{s}=\frac{k_{s}}{\sum_{s=1}^{n} k_{s}}, x_{\rho}=\frac{r_{s}}{k_{s}}, q_{s}=\frac{t_{u}}{\sum_{u=1}^{m} t_{u}}$, and $y_{s}=\frac{w_{u}}{t_{u}}$ in inequality (18) (for ositive weights), we have

$$
\sum_{s=1}^{n} \frac{k_{s}}{\sum_{s=1}^{n} k_{s}} f\left(\frac{r_{s}}{k_{s}}\right)-f\left(\sum_{s=1}^{n} \frac{r_{s}}{\sum_{s=1}^{n} k_{s}}\right) \leq \sum_{u=1}^{m} \frac{t_{u}}{\sum_{u=1}^{m} t_{u}} f\left(\frac{w_{u}}{t_{u}}\right)-f\left(\sum_{u=1} \frac{w_{u}}{m_{1}^{m} t_{u}}\right) .
$$

Multiplying (37) by the sum $\sum_{s=1}^{n} k_{s}$, we get

$$
\begin{array}{r}
\hat{I}_{f}(\tilde{\mathbf{r}}, \tilde{\mathbf{k}})-f\left(\sum_{s=1}^{n} \frac{r_{s}}{\sum_{s=1}^{n} k_{s}}\right) \sum_{s=1}^{n} k_{s} \leq \sum_{u=1}^{m} \frac{t_{u}}{\sum_{u=1}^{m} t_{u}} f\left(\frac{w_{u}}{t_{u}}\right) \sum_{k=1} k_{u} \\
f\left(\sum_{u=1}^{m} \frac{1}{\sum_{u=1}^{m}}\right) \sum_{s=1}^{n} k_{s} .
\end{array}
$$

Now again multiplying (38) by ne sum ${ }_{L}{ }_{1} t_{u}$, we get

$$
\begin{aligned}
& \sum_{u=1}^{m} t_{u} \hat{I}_{f}(\tilde{\mathbf{r}}, \tilde{\mathbf{k}})-f\left(\tau^{n} \frac{r_{s}}{\sum_{s=1}^{n} k_{s}}\right) \sum_{s=1} k_{s} \sum_{u=1}^{m} t_{u} \\
& \quad \leq \sum_{s=1}^{n} k_{s} \hat{I}_{f}-f\left(\sum_{u=1} \frac{w_{u}}{\sum_{u=1}^{m} t_{u}}\right) \sum_{s=1}^{n} k_{s} \sum_{u=1}^{m} t_{u} .
\end{aligned}
$$

If we $\mathrm{a}_{1} \quad$ ove inequality with the product $\sum_{s=1}^{n} k_{s} \sum_{u=1}^{m} t_{u}$, we get (35).

ii) Usin.$* i d f$ (where "id" is the identity function) in (18)(for positive weights), we hav

$$
\sum_{s=1}^{n} p_{s} x_{s} f\left(x_{s}\right)-\sum_{s=1}^{n} p_{s} x_{s} f\left(\sum_{s=1}^{n} p_{s} x_{s}\right) \leq \sum_{u=1}^{m} q_{u} y_{u} f\left(y_{u}\right)-\sum_{u=1}^{m} q_{u} y_{u} f\left(\sum_{u=1}^{m} q_{u} y_{u}\right) .
$$

Using the same steps as in the proof of (i), we get (36).

\subsection{Shannon entropy}

Definition 3 (see [9]) The $\mathcal{S}$ hannon entropy of positive probability distribution $\tilde{\mathbf{r}}=$ $\left(r_{1}, \ldots, r_{n}\right)$ is defined by

$$
\mathcal{S}:=-\sum_{s=1}^{n} r_{s} \log \left(r_{s}\right)
$$




\section{Corollary 6 Assume $\mathcal{G}$.}

$$
\begin{aligned}
& \text { If } \tilde{\mathbf{k}}=\left(k_{1}, \ldots, k_{n}\right) \in \mathbb{R}_{+}^{n}, \tilde{\mathbf{t}}=\left(t_{1}, \ldots, t_{m}\right) \in \mathbb{R}_{+}^{m} \text { and if base of } \log \text { is greater than } 1, \text { then } \\
& \frac{1}{\sum_{s=1}^{n} k_{s}}\left[\mathcal{S}+\sum_{s=1}^{n} r_{s} \log \left(k_{s}\right)\right]+\left[\sum_{s=1}^{n} \frac{r_{s}}{\sum_{s=1}^{n} k_{s}} \log \left(\sum_{s=1}^{n} \frac{r_{s}}{\sum_{s=1}^{n} k_{s}}\right)\right] \\
& \quad \leq \frac{1}{\sum_{u=1}^{m} t_{u}}\left[\tilde{\mathcal{S}}+\sum_{u=1}^{m} w_{u} \log \left(t_{u}\right)\right]+\left[\sum_{u=1}^{m} \frac{w_{u}}{\sum_{u=1}^{m} t_{u}} \log \left(\sum_{u=1}^{m} \frac{w_{u}}{\sum_{u=1}^{m} t_{u}}\right)\right]
\end{aligned}
$$

where $\mathcal{S}$ is defined in (39), and

$$
\tilde{\mathcal{S}}:=-\sum_{u=1}^{m} w_{u} \log \left(w_{u}\right)
$$

If base of $\log$ is less than 1 , then inequality (40) is reversed.

Proof The function $f \mapsto-x \log (x)$ is 3-convex for base of log 's gr ter than 1 . So, using $f:=-x \log (x)$ in Theorem 6(i), we get (40).

Remark 5 If $k$ and $t$ are positive probability distributions, the becomes

$$
\begin{aligned}
{\left[\mathcal{S}+\sum_{s=1}^{n} r_{s} \log \left(k_{s}\right)\right]+\left[\sum_{s=1}^{n} r_{s} \log \left(\sum_{s=1}^{n} r \mid \leq \tilde{S}^{\top}+\sum_{s=1}^{m} w_{s} \log \left(t_{s}\right)\right]\right.} & +\left[\sum_{s=1}^{m} w_{s} \log \left(\sum_{s=1}^{m} w_{s}\right)\right] .
\end{aligned}
$$

Definition 4 (see [9]) For $\hat{\mathbf{r}}$ and where $\tilde{\mathbf{r}}, \tilde{\mathbf{q}} \in \mathbb{R}_{+}^{n}$ the Kullback-Leibler divergence is defined by

$$
\mathcal{D}(\tilde{\mathbf{r}}, \tilde{\mathbf{q}}):=\sum_{s=1}^{n} n \log \left(\overline{q_{s}}\right) .
$$

\section{Coroll ry 7 Assun e $\mathcal{G}$.}

$\left.L_{\text {. }} \tilde{\mathbf{r}}=\ldots, r_{n}\right), \tilde{\mathbf{k}}=\left(k_{1}, \ldots, k_{n}\right) \in \mathbb{R}_{+}^{n}$, and $\tilde{\mathbf{w}}:=\left(w_{1}, \ldots, w_{m}\right), \tilde{\mathbf{t}}=\left(t_{1}, \ldots, t_{m}\right) \in \mathbb{R}_{+}^{m}$ be such $+\sum_{s=1}^{n} r_{s}, \sum_{s=1}^{n} k_{s}, \sum_{s=1}^{m} w_{s}$, and $\sum_{s=1}^{m} t_{s}$ be equal to 1 , then

$$
\sum_{s=1}\left(\frac{r_{s}}{k_{s}}\right) \mathcal{D}(\tilde{\mathbf{r}}, \tilde{\mathbf{k}})-\sum_{s=1}^{m}\left(\frac{w_{s}}{t_{s}}\right) \mathcal{D}(\tilde{\mathbf{w}}, \tilde{\mathbf{t}}) \geq 0
$$

where base of log is greater than 1.

If base of $\log$ is less than 1 , then the signs of inequality in (43) are reversed.

Proof In Theorem 6(ii), replacing $f$ by $-x \log (x)$, we have

$$
\begin{aligned}
& \frac{\sum_{s=1}^{n}\left(\frac{r_{s}}{k_{s}}\right)}{\sum_{s=1}^{n} k_{s}} \mathcal{D}(\tilde{\mathbf{r}}, \tilde{\mathbf{k}})-\sum_{s=1}^{n} \frac{r_{s}}{\sum_{s=1}^{n} k_{s}} \log \left(\sum_{s=1}^{n} \frac{r_{s}}{\sum_{s=1}^{n} k_{s}}\right) \\
& \geq \frac{\sum_{s=1}^{m}\left(\frac{w_{s}}{t_{s}}\right)}{\sum_{s=1}^{m} t_{s}} \mathcal{D}(\tilde{\mathbf{w}}, \tilde{\mathbf{t}})-\sum_{s=1}^{m} \frac{w_{s}}{\sum_{s=1}^{n} t_{s}} \log \left(\sum_{s=1}^{m} \frac{w_{s}}{\sum_{s=1}^{m} t_{s}}\right) .
\end{aligned}
$$


Now simply taking $\sum_{s=1}^{n} r_{s}, \sum_{s=1}^{n} k_{s}, \sum_{s=1}^{m} w_{s}$, and $\sum_{s=1}^{m} t_{s}$ are equal to 1 and after rearranging, we get (43).

\subsection{Rényi divergence and entropy}

The Rényi divergence and Rényi entropy are given in [19].

Definition 5 Let $\tilde{\mathbf{r}}, \tilde{\mathbf{q}} \in \mathbb{R}_{+}^{n}$ be such that $\sum_{1}^{n} r_{i}=1$ and $\sum_{1}^{n} q_{i}=1$, and let $\delta \geq 0, \delta \neq 1$.

(a) The Rényi divergence of order $\delta$ is defined by

$$
\mathcal{D}_{\delta}(\tilde{\mathbf{r}}, \tilde{\mathbf{q}}):=\frac{1}{\delta-1} \log \left(\sum_{i=1}^{n} q_{i}\left(\frac{r_{i}}{q_{i}}\right)^{\delta}\right)
$$

(b) The Rényi entropy of order $\delta$ of $\tilde{\mathbf{r}}$ is defined by

$$
\mathcal{H}_{\delta}(\tilde{\mathbf{r}}):=\frac{1}{1-\delta} \log \left(\sum_{i=1}^{n} r_{i}^{\delta}\right)
$$

These definitions also hold for nonnegative probability distr tions. If $\delta \rightarrow 1$ in (45), we have (42), and if $\delta \rightarrow 1$ in (46), then we have (39).

Now we obtain inequalities for the Rényi divergence.

\section{Theorem 7 Assume $\mathcal{G}$.}

Let $\left.\tilde{\mathbf{r}}=\left(r_{1}, \ldots, r_{n}\right), \tilde{\mathbf{k}}=\left(k_{1}, \ldots, k_{n}\right) \in \mathbb{R}_{+}^{n}, \tilde{\mathbf{w}}={ }_{1}, \ldots v_{m}\right)$, and $\tilde{\mathbf{t}}=\left(t_{1}, \ldots, t_{m}\right) \in \mathbb{R}_{+}^{m}$.

(i) If base of $\log$ is greater than 1 a $1 d 0 \leq \delta \leq$ ire such that $\delta, \theta \neq 1$, then

$$
\mathcal{D}_{\theta}(\tilde{\mathbf{r}}, \tilde{\mathbf{k}})-\mathcal{D}_{\delta}(\tilde{\mathbf{r}}, \tilde{\mathbf{k}})<\mathcal{D}_{\theta}\left(\tilde{\mathbf{w}}, \mathbf{\iota}, \mathcal{J}_{\delta}(\tilde{\mathbf{w}}, \tilde{\mathbf{t}}) .\right.
$$

If base of $\log$ is le, than 1, the inequality (47) holds in reverse.

(ii) If $\theta>1$ and if $b c$ of $\log$ is greater than 1 , then

$$
\mathcal{D}_{\theta}(\tilde{\mathbf{1}})-\mathcal{D}_{1}(\mathbf{r}, \mathbf{K}) \leq \mathcal{D}_{\theta}(\tilde{\mathbf{w}}, \tilde{\mathbf{t}})-\mathcal{D}_{1}(\tilde{\mathbf{w}}, \tilde{\mathbf{t}}) \text {. }
$$

(iii) If o 0,1$)$ and if base of $\log$ is greater than 1 , then

$$
\mathcal{D}_{1}(\tilde{\mathbf{r}}, \tilde{\mathbf{k}})-\mathcal{D}_{\delta}(\tilde{\mathbf{r}}, \tilde{\mathbf{k}}) \leq \mathcal{D}_{1}(\tilde{\mathbf{w}}, \tilde{\mathbf{t}})-\mathcal{D}_{\delta}(\tilde{\mathbf{w}}, \tilde{\mathbf{t}})
$$

Proo, Tith the mapping $f$ defined by $f:(0, \infty) \rightarrow \mathbb{R}$ by $f(t):=t^{\frac{\theta-1}{\delta-1}}$ and using

$$
p_{s}:=r_{s}, \quad x_{s}:=\left(\frac{r_{s}}{k_{s}}\right)^{\delta-1}, \quad s=1, \ldots, n,
$$

and

$$
q_{u}:=w_{u}, \quad y_{u}:=\left(\frac{w_{u}}{t_{u}}\right)^{\delta-1}, \quad u=1, \ldots, m,
$$

in (18) (for positive weights) and after simplifications, we have

$$
\sum_{s=1}^{n} k_{s}\left(\frac{r_{s}}{k_{s}}\right)^{\theta}-\left(\sum_{s=1}^{n} k_{s}\left(\frac{r_{s}}{k_{s}}\right)^{\delta}\right)^{\frac{\theta-1}{\delta-1}} \leq \sum_{u=1}^{m} t_{u}\left(\frac{w_{u}}{t_{u}}\right)^{\theta}-\left(\sum_{u=1}^{m} t_{u}\left(\frac{w_{u}}{t_{u}}\right)^{\delta}\right)^{\frac{\theta-1}{\delta-1}}
$$


if either $0 \leq \delta<1<\gamma$ or $1<\delta \leq \theta$, and inequality (50) holds in reverse if $0 \leq \delta \leq \gamma<1$. Raising the power $\frac{1}{\theta-1}$ in (50),

$$
\begin{aligned}
& \left(\sum_{s=1}^{n} k_{s}\left(\frac{r_{s}}{k_{s}}\right)^{\theta}\right)^{\frac{1}{\theta-1}}-\left(\sum_{s=1}^{n} k_{s}\left(\frac{r_{s}}{k_{s}}\right)^{\delta}\right)^{\frac{1}{\delta-1}} \\
& \leq\left(\sum_{u=1}^{m} t_{u}\left(\frac{w_{u}}{t_{u}}\right)^{\theta}\right)^{\frac{1}{\theta-1}}-\left(\sum_{u=1}^{m} t_{u}\left(\frac{w_{u}}{t_{u}}\right)^{\delta}\right)^{\frac{1}{\delta-1}} .
\end{aligned}
$$

For base of $\log$ is greater than 1 , the log function is increasing, therefore on takir $\mathrm{g} \log$ in (51), we get (47). If base of $\log$ is less than 1 , inequality in (47) is reversed. If $\delta=1 \quad \theta$, and by taking the limit, we have (48) and (49) respectively.

\section{Theorem 8 Assume $\mathcal{G}$.}

Let $\tilde{\mathbf{r}}=\left(r_{1}, \ldots, r_{n}\right), \tilde{\mathbf{k}}=\left(k_{1}, \ldots, k_{n}\right) \in \mathbb{R}_{+}^{n}, \tilde{\mathbf{w}}=\left(w_{1}, \ldots, w_{m}\right)$, and $\tilde{\mathbf{t}}, \ldots, t_{m},-\mathbb{R}_{+}^{m}$.

If either $1<\delta$ and base of $\log$ is greater than 1 or $\delta \in[0,1), \quad J b$ b f log is less than 1 , then

$$
\begin{aligned}
& \frac{1}{\sum_{s=1}^{n} k_{s}\left(\frac{r_{s}}{k_{s}}{ }^{\delta}\right.} \sum_{s=1}^{n} k_{s}\left(\frac{r_{s}}{k_{s}}\right)^{\delta} \log \left(\frac{r_{s}}{k_{s}}\right)-\mathcal{D}_{\delta}(\tilde{\mathbf{r}}, \tilde{\mathbf{k}}) \\
& \leq \frac{1}{\sum_{s=1}^{n} k_{s}\left(\frac{r_{s}}{k_{s}}\right)^{\delta}} \sum_{s=1}^{m} t_{s}\left(\frac{w_{s}}{t_{s}}\right)^{\delta} \log \left(\frac{w_{s}}{t_{s}^{t}} \sum_{s=s}^{n} \frac{\left(\frac{w_{s}}{t_{s}}\right)^{\delta}}{k_{s}\left(\frac{\left(\frac{r_{s}}{k_{s}}\right)^{\delta}}{\mathcal{D}_{\delta}}(\tilde{\mathbf{w}}, \tilde{\mathbf{t}}) .\right.}\right.
\end{aligned}
$$

If either $1<\delta$ and base of $\log i$ rreater. $n 1$ or $\delta \in[0,1)$ and base of $\log$ is less than 1 , inequality in (52) is reversed?

Proof The proof is on' for the case when $\delta \in[0,1)$ and base of $\log$ is greater than 1 , and similarly the remaining ses a simple to prove.

The function $x \quad f(x)(x>0)$ is 3-convex for base of $\log$ is less than 1. Also $0>\frac{1}{1-\delta}$ and choosing $I=(0, \alpha)$

$$
p_{s}:=x_{s}:=\left(\frac{r_{s}}{k_{s}}\right)^{\delta-1}, \quad s=1, \ldots, n,
$$

and

$$
q_{u}:=w_{u}, \quad y_{u}:=\left(\frac{w_{u}}{t_{u}}\right)^{\delta-1}, \quad u=1, \ldots, m,
$$

in (18) (for positive weights) and after simplifications, we have (52).

\section{Corollary 8 Assume $\mathcal{G}$.}

Let $\tilde{\mathbf{r}}=\left(r_{1}, \ldots, r_{n}\right), \tilde{\mathbf{k}}=\left(k_{1}, \ldots, k_{n}\right) \in \mathbb{R}_{+}^{n}, \tilde{\mathbf{w}}=\left(w_{1}, \ldots, w_{m}\right)$, and $\tilde{\mathbf{t}}=\left(t_{1}, \ldots, t_{m}\right) \in \mathbb{R}_{+}^{m}$ be such that $\sum_{s=1}^{n} r_{s}, \sum_{s=1}^{n} k_{s}, \sum_{u=1}^{m} w_{u}$, and $\sum_{u=1}^{m} t_{u}$ are equal to 1 .

(i) If base of $\log$ is greater than 1 and $0 \leq \delta \leq \theta$ such that $\delta, \theta \neq 1$, then

$$
\mathcal{H}_{\theta}(\tilde{\mathbf{r}})-\mathcal{H}_{\delta}(\tilde{\mathbf{r}}) \geq \mathcal{H}_{\theta}(\tilde{\mathbf{w}})-\mathcal{H}_{\delta}(\tilde{\mathbf{w}})
$$


The reverse inequality holds in (53) if base of $\log$ is less than 1.

(ii) If $1<\theta$ and base of $\log$ is greater than 1 , then

$$
\mathcal{H}_{\theta}(\tilde{\mathbf{r}})-\mathcal{S} \geq \mathcal{H}_{\theta}(\tilde{\mathbf{w}})-\tilde{\mathcal{S}}
$$

The reverse inequality holds in (54) if base of $\log$ is greater than 1.

(iii) If $0 \leq \delta<1$ and base of $\log$ is greater than 1 , then

$$
\mathcal{S}-\mathcal{H}_{\delta}(\tilde{\mathbf{r}}) \geq \tilde{\mathcal{S}}-\mathcal{H}_{\delta}(\tilde{\mathbf{w}})
$$

If base of $\log$ is less than 1, the inequality in (55) is reversed.

Proof (i) Suppose $\tilde{\mathbf{k}}, \tilde{\mathbf{t}}=\frac{\mathbf{1}}{\mathbf{n}}$. Then from (45) we have

$$
\mathcal{D}_{\delta}(\tilde{\mathbf{r}}, \tilde{\mathbf{q}})=\frac{1}{\delta-1} \log \left(\sum_{s=1}^{n} n^{\delta-1} r_{s}^{\delta}\right)=\log (n)+\frac{1}{\delta-1} \log \left(\sum_{s=1}^{n} r_{s}\right)
$$

and

$$
\mathcal{D}_{\delta}(\tilde{\mathbf{w}}, \tilde{\mathbf{t}})=\frac{1}{\delta-1} \log \left(\sum_{s=1}^{n} n^{\delta-1} w_{s}^{\delta}\right)=\log \left(r^{1}, 1 / \sum_{s=1}^{n} w_{s}^{\delta}\right) .
$$

We have

and

$$
\mathcal{H}_{\delta}(\tilde{\mathbf{r}})=\log (n)-\mathcal{D}_{\delta}\left(\tilde{\mathbf{r}}, \frac{\mathbf{1}}{\mathbf{1}}\right)
$$

$$
\mathcal{H}_{\delta}(\tilde{\mathbf{w}})=\log \quad \mathcal{D}_{\delta}\left(\tilde{\mathbf{w}}, \frac{1}{\mathbf{n}}\right) .
$$

We ge ${ }^{+}$53) fter us.ng Theorem 7(i), (56) and (57).

Scatem ts (11) and (iii) are similarly proved.

\section{Cor ary 9 Assume $\mathcal{G}$.}

Let $\tilde{\mathbf{v}}=\left(r_{1}, \ldots, r_{n}\right), \tilde{\mathbf{k}}=\left(k_{1}, \ldots, k_{n}\right), \tilde{\mathbf{w}}=\left(w_{1}, \ldots, w_{m}\right)$, and $\tilde{\mathbf{t}}=\left(t_{1}, \ldots, t_{n}\right)$ be positive probabi cty distributions.

If either $\delta \in[0,1)$ and base if $\log$ is greater than 1 , or $\delta>1$ and base if $\log$ is less than 1 , then

$$
-\frac{1}{\sum_{s=1}^{n} r_{s}^{\delta}} \sum_{s=1}^{n} r_{s}^{\delta} \log \left(r_{s}\right)-\mathcal{H}_{\delta}(r) \geq \frac{1}{\sum_{s=1}^{m} w_{s}^{\delta}} \sum_{s=1}^{m} w_{s}^{\delta} \log \left(w_{s}\right)-\frac{\sum_{s=1}^{m} w_{s}^{\delta}}{\sum_{s=1}^{n} r_{s}^{\delta}} \mathcal{H}_{\delta}(w) .
$$

The inequality in (58) is reversed if either $\delta \in[0,1)$ and base if $\log$ is less than 1 , or $\delta>1$ and the base of log is greater than 1.

Proof Proof is similar to Corollary 8 


\subsection{Zipf-Mandelbrot law}

In [14] the authors gave some contribution in analyzing the Zipf-Mandelbrot law which is defined as follows:

Definition 6 The Zipf-Mandelbrot law is a discrete probability distribution depending on three parameters: $\mathcal{N} \in\{1,2, \ldots\},, \phi \in[0, \infty)$, and $t>0$, and is defined by

$$
f(s ; \mathcal{N}, \phi, t):=\frac{1}{(s+\phi)^{t} \mathcal{H}_{\mathcal{N}, \phi, t}}, \quad s=1, \ldots, \mathcal{N}
$$

where

$$
\mathcal{H}_{\mathcal{N}, \phi, t}=\sum_{\nu=1}^{\mathcal{N}} \frac{1}{(v+\phi)^{t}} .
$$

For all values of $\mathcal{N}$, if the total mass of the law is taken, then $f \leq \phi, 1<\mathcal{C}, s \in \mathcal{N}$, the density function of the Zipf-Mandelbrot law becomes

$$
f(s ; \phi, t)=\frac{1}{(s+\phi)^{t} \mathcal{H}_{\phi, t}},
$$

where

$$
\mathcal{H}_{\phi, t}=\sum_{v=1}^{\infty} \frac{1}{(v+\phi)^{t}} .
$$

For $\phi=0$, the Zipf-Mandell rot - becomes Zipf's law.

Conclusion 1 Assum $\mathcal{G}$.

Let $\tilde{\mathbf{r}}$ and $\tilde{\mathbf{w}}$ be the $\mathrm{Zi}_{\mathrm{L}}$ Mardelbrot laws. By Corollary 8(iii). If $\delta \in[0,1)$ and base of $\log$ is greater than 1 ,

$$
\begin{aligned}
& \mathcal{L}-\frac{1}{(s+k)^{s} \mathcal{H}_{\mathcal{N}, k, v}} \log \left(\frac{1}{(s+k)^{s} \mathcal{H}_{\mathcal{N}, k, v}}\right)-\frac{1}{1-\delta} \log \left(\frac{1}{\mathcal{H}_{\mathcal{N}, k, v}^{\delta}} \sum_{s=1}^{n} \frac{1}{(s+k)^{\delta s}}\right) \\
& \geq \tilde{\mathcal{S}} \\
& =-\sum_{s=1}^{m} \frac{1}{(s+w)^{s} \mathcal{H}_{\mathcal{N}, w, v}} \log \left(\frac{1}{(s+w)^{s} \mathcal{H}_{\mathcal{N}, w, v}}\right)-\frac{1}{1-\delta} \log \left(\frac{1}{\mathcal{H}_{\mathcal{N}, w, v}^{\delta}} \sum_{s=1}^{m} \frac{1}{(s+w)^{\delta s}}\right) .
\end{aligned}
$$

The inequality is reversed if base of log is less than 1.

\section{Acknowledgements}

The authors wish to thank the anonymous referees for their very careful reading of the manuscript and fruitful comments and suggestions. The research of the 4th author is supported by the Ministry of Education and Science of the Russian Federation (the Agreement number No. 02.a03.21.0008).

Funding

There is no funding for this work. 
Authors' contributions

All authors jointly worked on the results and they read and approved the final manuscript.

\section{Author details}

'Department of Mathematics, University of Sargodha, Sargodha, Pakistan. ${ }^{2}$ Catholic University of Croatia, llica, Zagreb, Croatia. ${ }^{3}$ Rudn University, Moscow, Russia.

\section{Publisher's Note}

Springer Nature remains neutral with regard to jurisdictional claims in published maps and institutional affiliations.

Received: 14 March 2019 Accepted: 30 July 2019 Published online: 26 August 2019

\section{References}

1. Aras-Gazić, G., Čuljak, V., Pečarić, J., Vukelić, A.: Generalization of Jensen's inequality by Lidstone's polynomiz and related results. Math. Inequal. Appl. 164, 1243-1267 (2013)

2. Bullen, P.S.: An inequality of N. Levinson. Publ. Elektroteh. Fak. Univ. Beogr., Ser. Mat. Fiz. 109-112 (1973)

3. Cerone, P., Dragomir, S.S.: Some new Ostrowski-type bounds for the Čebyšev functional and applic. Inequal. 8(1), 159-170 (2014) 4. Csiszár, I.: Information-type measures of difference of probability distributions and indirect servations. via Sci.
Math. Hung. 2, 299-318 (1967)

5. Csiszár, I.: Information measures: a critical survey. In: Tans. 7th Prague Conf. on Info. Th., Statist. D Funct., Random Process and 8th European Meeting of Statist., vol. B, pp. 73-86. Academia, Prague ??

6. Diodato, V.: Dictionary of Bibliometrics. Haworth Press, New York (1994)

7. Egghe, L., Rousseau, R.: Introduction to Informetrics, Quantitative Methods in Li Information Science. Elsevier, New York (1990)

8. Gibbs, A.L.: On choosing and bounding probability metrics. Int. Stat. Re רח 419-435 ( 02 )

9. Horváth, L., Pečarić, Đ., Pečarić, J.: Estimations of f- and Rényi divergences u, a cyclic refinement of the Jensen's inequality. Bull. Malays. Math. Soc. 42(3) 933-946 (2019)

10. Khan, K.A., Niaz, T., Pečarić, Đ., Pečarić, J.: Refinement of Jensen's inequality ¿nd estimation of $f$ and Rényi divergence via Montgomery identity. J. Inequal. Appl. 2018, 318 (2018

11. Krnić, M., Lovričević, N., Pečarić, J.: Superadditivity of th evinso nctional and applications. Period. Math. Hung. 71(2), 166-178 (2015)

12. Levinson, N.: Generalization of an inequality of K, ann. J. I An il. Appl. 6, 133-134 (1969)

13. Liese, F., Vajda, I.: Convex Statistical Distances. Teubiner-Texte Mathematik, vol. 95. Teubner, Leipzig (1987)

14. Lovričević, N., Pečarić, Đ., Pečarić, J.: Zipf-Mar, $r$ ro...łw, divergences and the Jensen-type interpolating inequalities. J. Inequal. Appl. 2018, 36 (2sio)

15. Mercer, A.McD.: A variant of Jensen's ruality. J. Ir al. Pure Appl. Math. 4(4), article 73 (2003)

16. Mitrinović, D.S., Pečarić, J., Fink, A.M : Clà. \l and New Inequalities in Analysis, vol. 61. Kluwer Academic, Dordrecht (1992)

17. Pečarić, J.: On an inequality on N. Levinson. Py, ol. Elektroteh. Fak. Univ. Beogr., Ser. Mat. Fiz. $71-74$ (1980)

18. Popoviciu, T.: Sur une ined ite de N. Levinson. Mathematica 6, 301-306 (1969)

19. Rényi, A.: On measure of ir mation ar d entropy. In: Proceeding of the Fourth Berkely Symposium on Mathematics, Statistics and Probability, pp 7 - 56, (1960)

20. Sason, I., Verdú, S.. Divergence Inequalities. IEEE Trans. Inf. Theory 62, 5973-6006 (2016)

21. Vajda, I.: Theory of taur. Kerence and Information. Kluwer Academic, Dordrecht (1989)

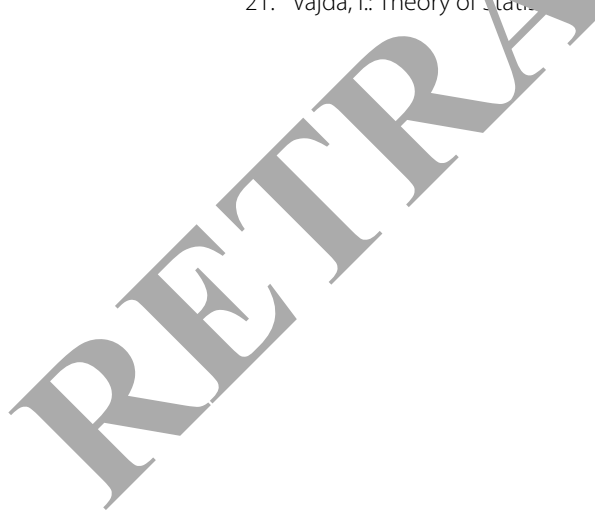

\section{Submit your manuscript to a SpringerOpen ${ }^{\circ}$ journal and benefit from:}

- Convenient online submission

- Rigorous peer review

- Open access: articles freely available online

- High visibility within the field

- Retaining the copyright to your article

Submit your next manuscript at $\gg$ springeropen.com 